which is presented has been arranged to be as near to that used in the scale-testing work as is practical.

It should be clearly understood that while the method was developed in connection with the testing of large weights, one of the fields of greatest usefulness is in the careful test of ordinary sized weights forming the working standards used by those testing scales and also for the test of counterpoise weights.

It is also believed that the publication will serve as a very useful reference for almost all industrial, engineering, or other technical laboratories where there is an occasional need for making weighings with more than ordinary accuracy. The paper offers a plan for undertaking such work promptly, as the steps to be taken and the record form and computation sheet to be used are presented in detail.

In the method of weighing by substitution presented in this publication readings are taken from the moving beam by means of a pointer and scale which are applied to the mechanism. The accuracy obtained is not limited by any error in the multiplication ratios of the weighing mechanism, but only by the precision with which it will repeat its indication.

The general matters covered by the paper comprise first a general description of the theory of weighing by substitution; the preparation of the scale for weighing so that the swings of the beam can be read on the graduated scale; the method for obtaining the positions of the equilibrium of the beam from the readings taken on it while moving; the method of removing and substituting weights ; and a description of the details to be observed in preparing the scale and making observations, and the practice to be followed in making the computations.

The method is of especial value in the calibration of a large number of weights of the same denomination. The method is equally applicable, however, for determining accurately the unknown weight of any object.

\title{
A Study of Franklin's Experiment on the Leyden Jar with
} Movable Coatings. G. L. AdDenbrooke. (Phil. Mag., March, 1922.) - The usual form of this experiment is as follows: A glass jar has an inner and an outer metallic coating, both closely fitting and capable of being removed. This jar is charged in the regular way. The inner coating is removed by an insulated holder. It then has 
scarcely any charge. It is touched to the outer coating which may be still in place or may have been separated from the glass. The two coatings are then put into position and it is found that the reconstituted jar has a strong charge. This is interpreted to mean that the seat of the charge was in the dielectric and that the charge remained there when the jar was taken apart.

The author of the paper could not reconcile the explanation with his views of electrical actions. He noted that glass was always mentioned as the material of the jar. He tried a jar of paraffin and found marked differences. Upon removal the inner coating had an electric charge. After the coatings had been touched and the jar put together, there was no appreciable charge left. Exactly the same results were obtained when precautions were taken to have the glass coatings free from moisture. It thus appears that in the experiment, as usually performed with glass as the dielectric, a damp layer on each side of the glass really serves as a coating. The removal of the coatings is, therefore, fictitious. The inner metal coating brings away with it only a little charge, the main part of the charge remaining behind on the damp layer.

It is suggested that the experiment be performed with the substitution of an ebonite or of a glass jar. The former should be kept in the dark with calcium chloride. The latter should be heated to $100^{\circ} \mathrm{C}$. and kept with the hygroscopic substance as long as possible.

G. F. S.

Natural Gas in I9r9. (U.S. Geological Survey Press Notice.)The production of natural gas in the United States in 1919 was II.8 per cent. more than in I9I8. The figures for I919, however, are not directly comparable with those for I9I8, because the production in IgIg includes the volume of gas wasted in the fields and in transmission, as far as reported by the operators. The quantity of gas wasted is the difference between the quantity produced and that actually consumed, a difference of $58,943,631,000$ cubic feet. As the figures for 1919 include this wasted gas, no value is given to the quantity produced. If the volume of gas wasted had been utilized it would have possessed a value of about $\$ 13,725,000$. This quantity does not include the gas wasted at the point of consumption. The quantity produced in I9I9, not including the gas wasted, is therefore the quantity consumed, which was 739,91 5,781,000 cubic feet, an increase of only 2.6 per cent. over I9I 8 .

The total acreage reported in I9I9 is considerably less than in I9I 8 and previous years because it includes only the acreage containing gas wells and that where gas wells predominate. The figures for past years included much of the oil acreage. It is impossible to scparate the natural gas and petroleum acreage. 